\title{
Development of Positron Timing Counter with SiPM Readout for MEG II Experiment
}

\author{
K. Yoshida ${ }^{* a}$, G. Boca ${ }^{c, d}$, P. W. Cattaneo ${ }^{c}$, M. De Gerone ${ }^{e}$, F. Gatti ${ }^{e, f}$, M. Nishimura ${ }^{a}$, \\ W. Ootani ${ }^{b}$, G. Pizzigoni ${ }^{e, f}$, M. Rossella $^{c}$, N. Shibata ${ }^{a}$, M. Simonetta ${ }^{c, d}$, and \\ Y. Uchiyama ${ }^{b}$ \\ ${ }^{a}$ Dep. of Physics, The University of Tokyo, Japan \\ ${ }^{b}$ ICEPP, The University of Tokyo, Japan \\ ${ }^{c}$ INFN Sezione di Pavia, Italy \\ ${ }^{d}$ Dip. di Fisica Nucleare e Teorica, Università degli Studi di Pavia, Italy \\ e INFN Sezione di Genova, Italy \\ ${ }^{f}$ Dip. di Fisica, Università degli Studi di Genova, Italy \\ E-mail: kyoshidadicepp.s.u-tokyo.ac.jp
}

The MEG experiment is now being upgraded to search for the charged lepton flavor violating decay $\mu^{+} \rightarrow e^{+} \gamma$ with an improved branching ratio sensitivity, $5 \times 10^{-14}$. We are developing a new Timing Counter to precisely measure the timing of the decay positrons under the high rate environment. The Timing Counter consists of 512 counters and each counter is based on a fast plastic scintillator plate readout by multiple SiPMs. The positron timing can be measured with improved time resolution by averaging positron impact times over multiple counter hits. We performed a beam test with a prototype to demonstrate the time resolution under a high rate environment. As a result, a timing resolution of $\sim 30$ ps was demonstrated at the expected positron rate. Furthermore, it is quite important to precisely time-align all the counters. We started to develop time calibration methods using a light pulse from a picosecond laser pulser distributed to all the counters. We tested the laser pulse injection to a single counter and measured the light amount, stability, and reproducibility, and the method is found to be promising for the precise time calibration in the MEG II experiment. The construction has been started and the part of timing counter will be installed in autumn 2015.

Flavor Physics \& CP Violation 2015

May 25-29, 2015

Nagoya, Japan

${ }^{*}$ Speaker. 


\section{Introduction}

The charged lepton flavor violating decay $\mu^{+} \rightarrow e^{+} \gamma$ has been searched for in the MEG experiment, setting the best limit on the branching ratio $\mathscr{B}<5.7 \times 10^{-13}$ [四]. It is now being upgraded to improve the sensitivity down to $5 \times 10^{-14}$ [D]. In the upgraded experiment, MEG II, the muon beam intensity will be doubled, $R_{\mu}=7 \times 10^{7}$ muons/s, and accordingly the accidental background will increase with $R_{\mu}^{2}$. Therefore, it is necessary to improve the resolutions of timing, position, and energy for positron and $\gamma$-ray.

In this paper, we focus on the upgrade of the positron Timing Counter (TC), which is devoted to measure the timing of the decay positrons. The new TC will be highly segmented with many small counters to achieve a $\sim 30$ ps time resolution. It is necessary to measure and prove the performance in an environment close to that of MEG II. The time alignment of different counters is also important for high granularity detectors.

\section{Upgraded Positron Timing Counter}

The basic concept of the upgraded TC is improving the time resolution down to $\sim 30$ ps by measuring the time of each positron using many counters. In the MEG experiment, the TC was composed of 30 scintillator-bars and PMTs for the readout. On the other hand, the upgraded TC consists of 512 small counters with SiPM readout as shown in Figure 1.

Each of the counters consists of $120 \times 40 \times 5$ or $120 \times 50 \times 5 \mathrm{~mm}^{3}$ fast plastic scintillator plate (BC422) and 6 AdvanSiD near-ultraviolet sensitive SiPMs connected in series attached on each end (Figure 2). The 2 sizes counters are properly arranged in view of the positron trajectory to raise the acceptance. The counters are mounted on 1-m long PCBs, which transmit the counter signal to the end of the detector via the coaxial-like signal line. Then the signals are transmitted on coaxial cables to the waveform digitizer WaveDREAM [3], which includes a voltage amplifier and a shaper performing pole-zero cancellation. For the details of the counter design and the optimization study see [四][回].

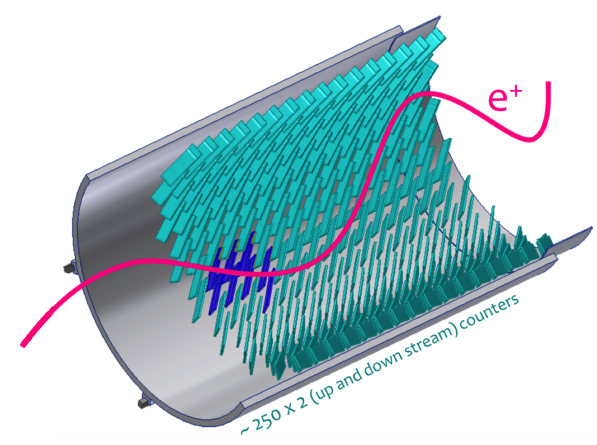

Figure 1: Upgraded timing counter overview. The scintillator and SiPMs (double-end readout). Scintilred line shows a signal positron trajectory in the MC lator is wrapped in refrector. simulation. The positron hits several counters.

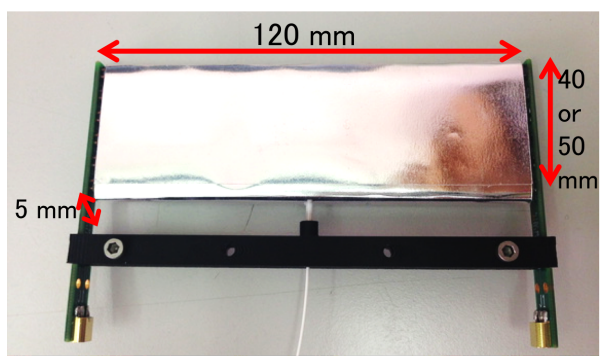

Figure 2: Picture of a counter. Counter consists of 


\section{Beam Test}

We performed a beam test with the prototype to demonstrate the expected time resolution in a high rate environment under MEG II beam condition in autumn 2014.

The prototype consists of 8 counters aligned so that the configuration is similar to the final TC. However, different scintillator (EJ232) and SiPMs with different characteristics (ASD-NUV3S-P50 ), which was found to have worse timing resolution than the final product, were used in this beam test. The $\pi \mathrm{E} 5$ beam line in PSI was used to provide $\sim 10^{8} \mathrm{~Hz}$ continuous muon beam. The muon beam is collimated by a collimator system and stopped in a plastic target. The Michel positrons from the target were measured by the prototype. The time reference and the trigger were made from 2 reference counters set in front of the prototype. We took data at 3 beam intensities resulting in 3 conditions of the positron hit rate: lower rate, expected rate, and higher rate than that expected in MEG II. However, the hit rate is dependent on the counter position, and hence, the actual hit rates are different for the counters.

Figure 3 shows the results of the time resolution at different positron hit rates. The time resolution is calculated from the sigma of the difference between the average time of hit counters and the reference time with subtraction of the reference counter time resolution. We achieved the target time resolution of $\sim 35 \mathrm{ps}$ at 8 counters hits at the expected positron rate in MEG II (the average rate: $70.2 \mathrm{kHz}$, the highest rate: $174 \mathrm{kHz}$ by the $\mathrm{MC}$ simulation). Also, the pile up effect turned out to be small for the time resolution. The time resolution is expected to further improve for the final detector with better the single counter resolution, $65 \mathrm{ps}$ instead of 90 ps obtained in this test.
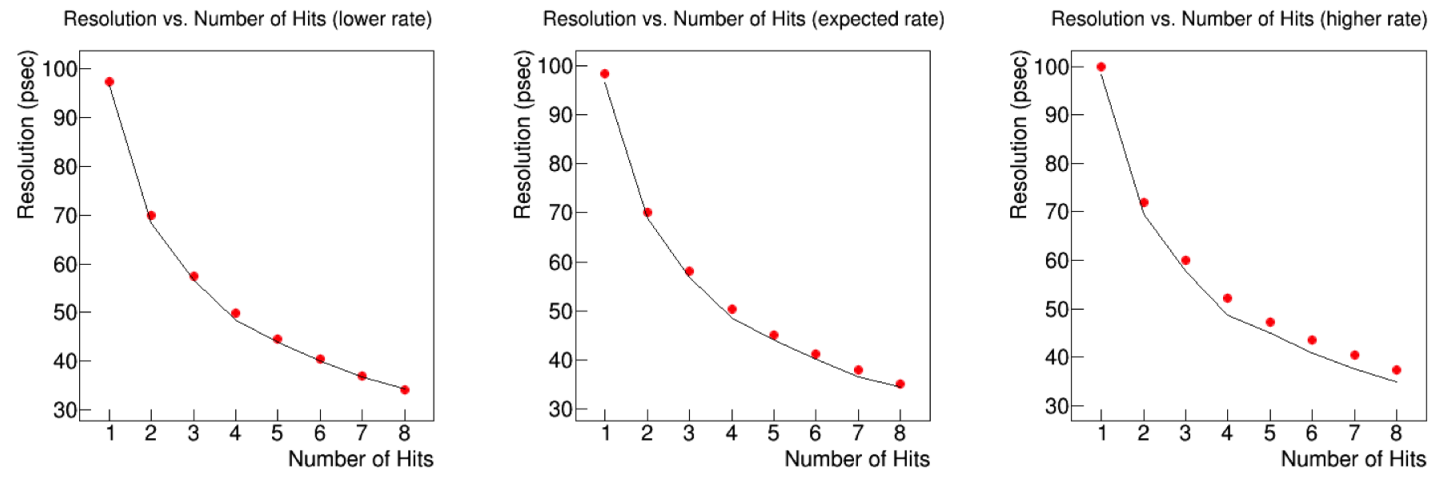

Figure 3: Overall resolution vs number of hit counters under the 3 conditions. Left plot is at lower rate $(17.8-64 \mathrm{kHz})$. Center plot is at the expected rate $(53-166 \mathrm{kHz})$. Right plot is at higher rate $(89-290 \mathrm{kHz})$. Resolution is improved with the number of hit counters. Pile up effect is marginal at the expected rate.

\section{Laser Calibration Study}

Each of the counters has a different time offset caused by the difference of electronics and inherent counter property. Therefore, it is necessary to calibrate the relative time offset to reconstruct positron timing correctly. We have already developed a method using Michel positrons from $\mu^{+} \rightarrow e^{+} v \bar{v}$. In this method, positrons crossing multiple counters are exploited to calibrate and monitor time offsets during the data taking with an expected resolution for neighbouring counters 
of $\sim 5$ ps as estimated by MonteCarlo simulation. However, the measurement may be affected by the hit position and the counters of upstream and downstream sectors cannot be calibrated at the same time. Therefore, we started to develop another calibration method by using laser pulses to complement for the Michel method. In this method, all counters can be calibrated simultaneously even in advance. The target precision is RMS 30 ps.

Figure 4 shows the basic concept of the laser calibration system. The common light from a laser pulser is divided by a optical switch and optical splitters and injected to 512 counters through optical fiber. The optical switch can switch the light by moving optical fiber using a piezoelectric actuator. It is used to obtain sufficient light intensity. The optical splitter splits the light using divided optical fibers by melt-drawing. The laser light is split into 64 channels by two step optical splitters of $1 \times 8$. Multimode optical fiber $(50 \mu \mathrm{m} \phi)$ is used because a transmission loss of singlemode fiber is high. The variance of multimode light was measured and is sufficiently small for our measurement. A mode scrambler is also used to uniform the light mode because the light has multi modes in optical fiber. We started the development to realize the laser calibration system.

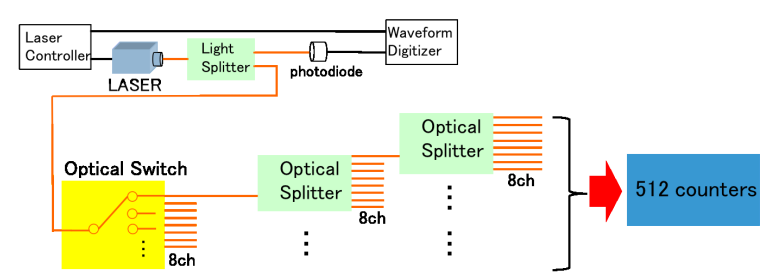

Figure 4: Concept of Laser calibration system. Laser light is divided to 512 counters. A photodiode is used as monitor for the laser light.

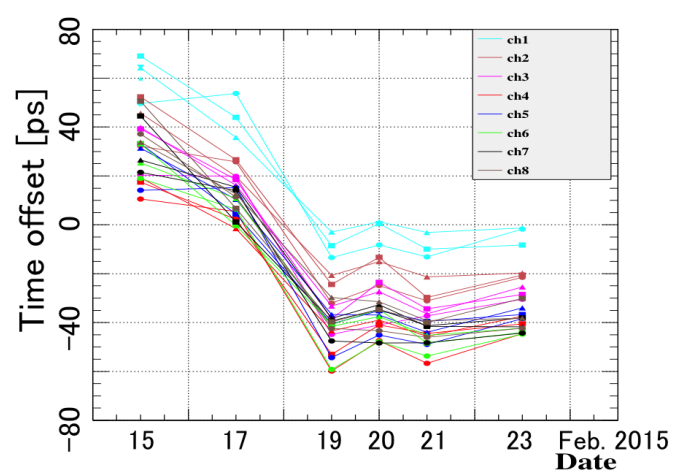

Figure 5: Time offset for measurement date at each optical splitter channel.

We measured the time offset of one counter into which the laser light is injected to investigate the detected amount of light, the stability and, the reproducibility. In this test, the laser light goes to the mode scrambler and 2 stages of the light splitters and the counter. The counter and the optical fiber are coupled with optical grease. The laser source is Pico second Light Pulser PLP10 (Hamamatsu). The wavelength is $401 \mathrm{~nm}$, close to the scintillation light. The pulse width is FWHM 60ps and the jitter is sufficiently small. The pulse frequency is tunable within 10-100 MHz and the peak power is $200 \mathrm{~mW}$. The synchronized signal from the laser controller is used for the trigger. The time offset is defined as the mean of a difference between the signal time from counter and the synchronization signal time. The measurement precision (statistical) of the time offset is high $(<1 \mathrm{ps})$.

By the measurement of time offset, we confirmed a good amount of light detected, laser stability and excellent reproducibility (RMS: $\sim 5 \mathrm{ps}$ ). Therefore, the laser calibration system is promising for the actual TC. However, shift of the time offsets was observed during repeat measurements (Figure 5). It may be caused by connection instability of the optical fiber and counter. It is under study 
for the connection method. Also, different time offsets among splitter channels were observed. However, constant time offsets can be corrected by calibrating them in advance. We will test the multi counter application toward the final TC.

\section{Summary}

We are upgrading the positron timing counter for the MEG II experiment. The upgraded TC is pixelated by 512 small counters consisting of a fast plastic scintillator and SiPMs readout. The time resolution of $\sim 35$ ps was demonstrated in the beam test under MEG II beam condition. The time calibration methods are under development and the study of the laser calibration system has progressed. Thereby, the counter design was decided and mass test has been started. We will install and test half of the Timing Counter (downstream side 256 counters) on the muon beam later this year.

\section{References}

[1] J. Adam et al., Phys. Rev. Lett. 110, 201801 (2013).

[2] A. M. Baldini et al., arXiv:1301.7225 (2013).

[3] S. Ritt et al., presented at 13th Pisa Meeting on Advanced Detectors (2015).

[4] P. W. Cattaone et al., IEEE Trans. Nucl. Sci. 61, 2657-2666 (2014).

[5] M. De Gerone et al., J. Instrm. 9, C02035 (2014). 